Voix et Images

volxetimages

\title{
Pierre Perrault. L'homme et sa parole
}

Jocelyne Tessier

Volume 3, numéro 3, avril 1978

Pierre Perrault

URI : https://id.erudit.org/iderudit/200118ar

DOI : https://doi.org/10.7202/200118ar

Aller au sommaire du numéro

Éditeur(s)

Les Presses de l'Université du Québec

ISSN

0318-9201 (imprimé)

1705-933X (numérique)

Découvrir la revue

Citer cet article

Tessier, J. (1978). Pierre Perrault. L'homme et sa parole. Voix et Images, 3(3),

379-395. https://doi.org/10.7202/200118ar

Ce document est protégé par la loi sur le droit d'auteur. L'utilisation des services d'Érudit (y compris la reproduction) est assujettie à sa politique d'utilisation que vous pouvez consulter en ligne.

https://apropos.erudit.org/fr/usagers/politique-dutilisation/
Cet article est diffusé et préservé par Érudit.

Érudit est un consortium interuniversitaire sans but lucratif composé de l’Université de Montréal, l'Université Laval et l'Université du Québec à Montréal. Il a pour mission la promotion et la valorisation de la recherche. https://www.erudit.org/fr/ 


\section{Pierre Perrault \\ L'homme et sa parole}

L'œuvre poétique de Pierre Perrault lui a mérité déjà quelques grands honneurs'. Les hommages à son talent de poète marquent même sa renommée en tant que cinéaste et dramaturge. Si Perrault n'a pas écrit de drames tout récemment, il vient de publier un nouveau recueil de poèmes intitulé Gelivures $^{2}$; et ses trois premiers volumes, Portulan (1961), Ballades du temps précieux (1963) et En désespoir de cause (1971), sont réédités en un seul livre, Chouennes ${ }^{3}$. C'est l'occasion rêvée pour mettre en évidence une facette de la créativité de Pierre Perrault qu'on a remarquée dans toutes ses œuvres artistiques.

La poésie de Perrault se distingue par les merveilles qu'elle révèle: merveilles de la mer et de la toundra; merveilles de la nature primitive des hommes et des bêtes; merveilles des principes universels qui gèrent l'ordre du monde. Le poète décrit avec enthousiasme l'immensité, le charme, les beautés, les richesses et les rigueurs de la mer qu'est le fleuve et de la toundra qu'est l'Abitibi. II raconte avec fascination les oiseaux, les loupsmarins, les dauphins, les bœufs musqués. Mais il chante d'abord et avant tout, la grandeur des bûcherons et des marins, des chasseurs et des pêcheurs; il célèbre la sagesse, l'héroïsme et l'habileté de ce peuple méconnu, race d'exilés et d'éternels voyageurs, qu'il a découvert tout au long de sa vie de scripteur et de cinéaste. Enfin, les découvertes régionalistes du poète débouchent sur des considérations transcendantes à travers l'exploitation verbale de tout un réseau de symboles. Par ses héros et ses merveilles, la poésie de Pịerre Perrault rejoint les grands schèmes universels, caractéristiques des œuvres mythiques, et, elle a cela de particulier, d'être de son temps et du Québec.

Avant d'essayer de préciser ce qui illustre le style de Perrault, j'aimerais présenter brièvement ses publications poétiques: Toutes is/es ${ }^{4}$, chroniques de terre et mer; Chouennes; Gélivures; les poèmes Noël ancien dans une vallée nouvelle ${ }^{5}$ et Chanson des voyageurs du temps passe en vain $^{6}$; et les deux textes intitulés $J$ 'habite une ville? 


\section{Toutes isles}

Toutes isles n'est pas un recueil de poèmes; c'est une étude anthropologique ennoblie par le souffle de la poésie. La série de récits, "poèmes en prose aussi beaux que les vers de Portulan ${ }^{8} "$, établit un lien entre la vie du cinéaste et ses poèmes: Toutes isles transpose, en paroles fabuleuses, les images des films, Au pays de Neufve-France. En cinq parties d'inégale longueur, l'auteur, inspiré par les chroniques de Jacques Cartier, reprend, au $x^{e}$ siècle, le voyage du célèbre explorateur: il décrit le village de Tête-à-la-Baleine qui quitte la terre ferme et s'installe sur l'île à l'été; il raconte la pêche miraculeuse, la vie nomade des habitants des îles, la mort d'un homme, le déménagement d'une maison de l'île à la terre ferme ; il suit les rites de la chasse au loup-marin et les gestes de la fonderie; et enfin, il consacre son plus long chapitre à la vie des Montagnais d'Olomanshibou. La vie des gens de la Côte Nord, transmise dans Toutes isles par illustration verbale seulement, prend une allure épique.

Dans le cadre de la nature sauvage se déroulent des exploits merveilleux. Les bêtes et les hommes vivent au rythme des saisons et de cette terre qu'ils ont choisie. La pêche et la chasse se présentent comme des tragédies de l'antiquité. La banalité quotidienne de la vie des pêcheurs de morues est travestie en drame avec des réminiscences de vie de pirates et des souvenances des trois navires de Saint-Malo. Sous la plume de Perrault, le cycle mystérieux de la vie rappelle les rites sacrés à l'origine des Grandes Dionysies. Telle est la description des morues, des loupsmarins et des marsouins qui poursuivent les capelans attirés par la lune de juin :

Ce spectacle inquiétant, élevé, magique, trouble l'eau ineffable de sauts, de rapts et d'encens tenace... indiquant la pierre des sacrifices ${ }^{9}$.

Et les gestes des hommes se mêlent aux rites de la nature: le poète assiste au "rituel des filets", à "la gigue du Labrador" qui conjure le froid, au drame obscur des déchargements de nuits à Olomanshibou; il parle du chant prophétique du makoucham et de la danse envoûtante du retour de chasse.

"Aucun écrivain canadien-français, je pense, n'est venu aussi près que Pierre Perrault, de faire surgir de notre pays un grand mythe ${ }^{10}$ ", écrit Gilles Marcotte. Et les critiques s'entendent pour retrouver dans Toutes isles l'envoûtement des épopées antiques et des grands livres de merveilles qui plongent l'homme 'dans le monde mystérieux de ses origines primitives.

\section{Chouennes}

Les Éditions de l'Hexagone ont publié Chouennes dans leur collection «Retrospectives": la présentation est aérée, simple et claire. Le poète 
a profité de cette publication qui inclut aussi trois Inédits, pour retoucher certains textes de ses premières éditions. L'ordre des poèmes a été remanié dans Portulan et Ballades du temps précieux pour un meilleur équilibre dans la disposition des textes. Les passages ajoutés, retranchés ou reformulés sont tout à l'avantage de la nouvelle édition. Pierre Perrault explique dès le début son choix du mot "Chouennes" comme titre du recueil: dans le langage des gens de Charlevoix, ce mot

recouvre toutes formes de
langage parlé, de la simple
vantardise à tout discours habile
à "dire des merveilles"...
"l reste qu'il me convient
d'en abuser pour ce qu'il contient
de vantardise et pour ce qu'il présage
un avenir à une parole qui
n'attend rien des hommes de loi
pour prendre place au soleil"1.

Cette fierté, pleine d'amour à l'égard des gens de la Côte Nord et de leur parlure en particulier, Perrault l'a affichée à travers toute son cuvre qui est en vérité une entreprise d'orgueil à la gloire du peuple québécois.

Chouennes s'ouvre sur Portulan qui est à la fois un regard émerveillé, sous l'emprise de la découverte d'un espace et des hommes qui l'habitent, et une vision inquiétante devant le silence et l'oubli, sombres présages de la disparition d'un peuple. Le mot «portulan" réfère à un livre de marin du $x v^{e}$ siècle où étaient décrits les ports, les côtes, les courants et les marées; ce livre servait de guide aux navigateurs pour reconnaître les points de repères, les amers, qui leur indiquaient la route le long des côtes. Pierre Perrault justement veut fixer les amers de son monde intérieur: il a découvert un pays mais il le décrit ici sans jamais le nommer. Les textés datent tout probablement de l'époque où Perrault était scripteur à Radio-Canada: au temps des émissions radiophoniques (1955-1960) et des films $A u$ pays de Neufve-France. Les poèmes de son premier recueil sont courts et nombreux. Les symboles s'entrelacent, s'opposent, se recoupent: la nuit, image du passé, du silence et de l'oubli appelle le symbolisme du jour où le soleil rejoint les images du verger; autour de l'idée du. verger, du jardin, se rassemblent les allusions aux arbres, aux fruits, aux fleurs et aux oiseaux. Ces images se mêlent à celle du voyage avec ses départs et ses retours et à celles de la femme identifiée à la terre, à la race ou à la langue. Et tout ce symbolisme illustre les thèmes de la parole, de l'appartenance, de l'amour, de la liberté, de la fuite du temps, thèmes universels du lyrisme qui ne rejoignent pas uniquement des préoccupations québécoises. Le poème Familier, par exemple, genre d'ode bucolique, invite à la fierté d'être soi-même, d'être simple, sans artifices; le poème fantaisiste Pour dormir explique à la terre le mystère de la pluie qui lui invente des miroirs; Autrefois déjà imagine avec tendresse les derniers jours d'un vieux couple... Pourtant on ne peut lire Naguère, Transhumance ou Promenade sans penser aux gens de la Cote Nord, aux chasseurs de caribous, 
aux capitaines de goélettes; on ne peut lire le Sang des muets, Fruit défendu, À poing fermé sans reconnaître l'intérêt du poète dans le sort des hommes de son pays.

Les poèmes de Ballades du temps précieux viennent en deuxième partie dans Chouennes. Ces poèmes remontent à l'époque des émissions radiophoniques du même nom et du premier long métrage de Perrault, Pour la suite du monde. L'attitude du poète est celle de l'observateur d'événements et du chantre des gestes qui durent. Le mot “pays» est employé. Le nom de "Labrador" paraît en titre. Tout ce que Pierre Perrault décrit retrace l'évidence de merveilles inouïes, de mystères essentiels ou de légendes immémoriales, comme l'histoire de l'Escargot pèlerin:

il a laissé dans la pierre traces de ses architectures toute matière n'est que sécrétion du recueillement fondamental... et la trace d'un encornet éloigne le commencement de la fin nous laissant perplexes ${ }^{12}$

Là où l'on aurait cru la vie absente, Perrault découvre une activité secrète et plus que millénaire (Marécage). Pourtant ce qu'il décrit conserve un aspect familier même sous les formes les plus énigmatiques. Chaque poème se rattache de près ou de loin à un aspect du pays ou de la vie du peuple québécois: Outarde, par le souvenir de la chasse aux canards; Nuit blanche par l'allusion au culte du passé, «ce temps / s'il revenait / dont nous aurions peur "; Plus que naître, par l'allégorie de la naissance d'un peuple.

En troisième partie, Chouennes présente En désespoir de cause, publié après les événements d'octobre 1970, après les enlèvements de Cross et de Laporte et l'imposition des mesures de guerre. Ces "poèmes de circonstances atténuantes" cherchent à expliquer les gestes d'Octobre. La nouvelle édition conserve les cinq divisions originales d'En désespoir de cause. D'une verve essoufflante, la première section, le Plus que temps, contient cinq longs poèmes en prose où s'intercalent de brèves strophes en vers libres: $\grave{A}$ bout de patience exprime la colère du poète qui parle au nom de la collectivité frustrée dans son désir de pays; le Fer au feu. réclame le pays; la Malengueulée dénonce les injustices et les misères; À vrai dire prend la part de ceux qui parlent «joual"; et enfin, le Cantouque des cantouques, variation du Cantique des cantiques de la Bible, rappelle le titre d'un recueil de poèmes de Gérald Godin et le terme marin pour désigner une sorte de grappin, mais c'est avant tout l'exaltation de l'amour d'un Québécois ordinaire pour son pays troublé. Cette première section, le Plus que temps, est en quelque sorte une longue déclaration d'amour: Perrault, parle de «prétentions territoriales" comme on parle de prétentions matrimoniales, à l'endroit de cette "terre d'élec- 
tion»; il déclare son amour "sans bon sens» pour ce pays qu'il nomme “québécoisie». Ce grand pays, ce corps immense, est animé par une âme, un esprit, un peuple qui, dans le Cantouque des cantouques devient pour le poète sa “lumineuse moitié d'ombre». La deuxième section, Septémbre contient quatre poèmes: Bilan provisoire, Éventail et À titre d'oiseau expriment de façon laconique quelques réflexions du poète qui ne s'illusionne pas; Déclaration de guerre, en six pages, révèle son acharnement à «tirer de l'oubli " ce pays qu'il aime. La troisième section, Octobre, n'est qu'une seule phrase: une infinitive qui résume le but des paroles et des gestes de l'heure - «rendre le futur disponible à ceux qui n'ont pas d'histoire " et une constatation "je n'en sortirai pas vivant de ce poème à outrance". La quatrième, Novembre, contient onze poèmes: Témoignage et Parler d'octobre renvoient directement aux antagonistes des enlèvements de l'automne 1970; les cinq poèmes suivants soulignent le besoin de parler pour la collectivité réduite au silence; Mon trère le désespoir et En toute innocence font l'apologie des felquistes; Ensuite, ma mie rappelle le malaise d'après la crise et Berceuse pour enfants perdus d'avance au-reste du monde évoque les héros de 1760 et 1837, Lévis, Papineau, Chénier, les poètes d'aujourd'hui, DesRochers et.Miron, et le métis Riel. La dernière section d'En désespoir de cause, Pour Noël, est un souhait: prévoyant avec cynisme, que le gouvernement rapatriera les investisseurs étrangers en révoquant les mesures de guerre, Perrault souhaite à tous « un joyeux Québec!» II est évident que ce recueil colle davantage à l'actualité. Les poèmes, comparés à ceux des deux premiers recueils, sont plus prosaïques, farcis d'expressions populaires; ils relèvent parfois plus de l'éloquence et de la diatribe que de la poésie. L'emploi d'un vocabulaire juridique fait image dans l'alternance de plaidoyers et de réquisitoires: le poète se voit incriminé, se sent «coupable d'espoir»; il veut qu'on reconnaisse la légitimité du combat; il parle de la clandestinité du Québécois; il reproche aux lois d'abuser de pouvoir. En désespoir de cause exploite un autre langage qui n'a pas souvent eu cours en poésie: un vocabulaire du domaine commercial donne un ton vraiment nord-américain et "vingtième siècle" aux envolées lyriques de ces derniers poèmes. Perrault «négocie avec la vengeance des berceaux", «défie les ordinateurs" et se "paie de mots à défaut de retirer des prestations d'ignorance»; il a le goût de "majorer la parole interdite"; "la vie m'endosse. comme une traite" écritil, et la guerre des prix comme un combat de dinosauriens, écrase les gagne-petit." Enfin un vocabulaire martial met en relief la virulence, l'ironie, le cynisme et l'impatience caractéristiques d'En désespoir de cause: “Depuis ma connaissance, je cultivais le sentiment d'être occupé, assiégé, investi, cerné, prisonnier, assailli, battu à plate couture»; Perrault veut "renverser le régime du silence"; il s'arme de "folies à répétitions". Sa violence est verbale: il parle de ses «fusillades vernaculaires", et de ses "armes blanches à l'âge atomique". Le rythme, accéléré par les énumérations, les répétitions, les pléonasmes et l'absence de ponctuation; ajoute à l'impression de colère inapaisable. 
Chouennes contient aussi trois textes que l'auteur choisit d'appeler Inédits. Trumeaux, poème de circonstance écrit pour accompagner un dessin, parle de miroirs oubliés dans les greniers et inspire un désir de prendre conscience de soi, de se regarder bien en face; le genre rappelle certains poèmes de Portulan. Un poisson dans la mer est paru, dans une première version, en préface à un recueil de poèmes d'étudiantes, en Belles-Lettres au Collège Notre-Dame, à Montréal, en 1962-1963. En dix ans, le texte est passé d'une centaine de mots à plus d'une douzaine de pages. Un poisson dans la mer, tel que publié dans Chouennes, est un hymne à la parole, comparable par son style au Cantouque des cantouques, ce poème d'amour du pays. Le poète y parle de délivrance, de la naissance d'une langue, de l'espoir de créer et de nommer un pays. On y retrouve l'emploi de termes juridiques, commerciaux et guerriers dans la dénonciation des linguistes, des juristes, des épiciers vendeurs de cidre et des ennemis de la "parole sauvage confiée aux fruits défendus". Le dernier texte du recueil, la Chanson de Marie n'est pas vraiment un inédit. Écrite avant 1967, à l'époque du film le Règne du jour, la chanson paraît dans la transcription du film publiée par Lidec; elle prend une intensité particulière quand elle est mise en musique, synchronisée aux images du film et insérée peu à peu, à trois endroits au cours du long métrage. Dans Chouennes, quand on ne connaît pas les héros du film, Marie Tremblay et son époux Alexis, la chanson prend une dimension plus universelle avec ses allusions au temps qui passe, aux souvenirs d'une vie simple et d'un grand amour, à l'approche de la mort.

Trumeaux, la Chanson de Marie, les poèmes de Portulan et de Ballades du temps précieux ressemblent davantage par la facture des vers, par la disposition des strophes et même par l'imagerie, aux plus traditionnels des poèmes modernes de la dernière moitié du $x x^{e}$ siècle. Les poèmes se limitent à des formules d'expression qui leur donnent un ton plus universel. Mais dès ses premiers recueils, Perrault utilise certains procédés littéraires qu'il va toujours affectionner: le goût du calembour et de l'aphorisme, les rapprochements inusités de clichés, une terminologie marine recueillie le long du fleuve, des allusions liturgiques et folkloriques, et les effets rythmiques de la répétition. Les deux premiers recueils recèlent aussi les préoccupations dominantes du poète; avant tout, l'amour des gens du pays et du temps présent puis la peur de perdre une identité précieuse, la méfiance de l'attachement aux choses d'un temps révolu, la lutte contre le silence, l'indifférence, l'inconscience, et enfin, le respect de la parole, ultime espoir de vaincre la mort d'un peuple. Un poisson dans la mer et les poèmes d'En désespoir de cause prennent la liberté d'employer des régionalismes, de citer des noms de personnes et de lieux, de rappeler des événements très précis, de créer des néologismes et d'exploiter un vocabulaire qu'on ne remarque pas dans les premiers recueils. Dans l'ensemble, Chouennes marquent ainsi l'évolution d'une poésie qui mérite d'être lue et relue. 


\section{Gélivures}

Depuis plus de dix ans, Pierre Perrault rédige ce qui pourrait devenir un très long poème épique en deux volumes. L'œuvre est chargée de réminiscences de ses films, surtout des plus récents: Un Royaume vous attend et Mouchouanipi. Le premier volet vient d'être publié par les éditions de l'Hexagone dans un format semblable au recueil Chouennes. Mais le nouveau recueil, intitulé Gélivures, a ceci de particulier qu'il présente un ensemble de trois parties qui s'enchaînent et qu'il faut lire l'une à la suite de l'autre pour conserver tout l'impact de la progression dramatique. Oh! il n'est pas impossible de lire Cornouailles avant Neigeries mais c'est préférable de suivre l'ordre imposé par le poète.

Neigeries, comme "rêveries", est le titre de la première section: I'histoire d'un rêve de royaume, fondée sur l'épique colonisation de l'Abitibi et la lutte des derniers fils de ces pionniers, est enrichie des leçons du lichen et des Inuits.

Froidureté, la deuxième section du recueil, offre en exemple le sort du chasseur autochtone pour mieux mettre en valeur les prétentions du poète à dire cette "présence des hommes au pays des hommes" et à parler de leurs rêves et du grand désir de royaume.

Enfin, Cornouailles établit un parallèle entre un combat d'ovibos et la situation du Québec: la harde convoitée, "femme entre toutes les neiges", symbolise à la fois le rêve, le royaume, le pays et la langue:

et sans autre but que la victoire

nous avons ma laineuse

entrepris ce long corps à corps perdu ${ }^{13}$

Pierre Perrault a hésité entre le titre Gélivures et $\dot{A}$ couteaux tirés, c'est-à-dire, en guerre ouverte contre "l'injuste milieu" et "le temps perdu". Il a choisi Gélivures qui désigne selon lui, "l'action du froid sur l'écorce des arbres et des hommes". Le mot illustre mieux d'ailleurs toute l'ampleur de l'épopée du peuple qu'il veut célébrer. Le poète a recueilli le mot "gélivures" dans le livre l'Homme et l'hiver au Canada du Français Pierre Deffontaines. Ce dernier écrit: “Nombre d'érables ou de hêtres sont zébrés de craquelures, que les Canadiens français appellent joliment des gélivures ${ }^{14}$." Perrault a cru qu'il s'agissait d'un canadianisme jusqu'à ce qu'il se rende à l'évidence du dictionnaire. Mais peu importe, le mot avait fait son chemin et incarnait déjà toute une symbolique du froid dans la perspective poétique de Perrault.

Le style de Gélivures rappelle par moment la verve frénétique d'En désespoir de cause; la poésie est sertie de citations de poètes, de scientifiques et d'explorateurs, comme Anne Hébert, Willy Ley et Jacques Cartier, et parsemée de récits et d'allusions à des histoires vécues comme celle du cheval ombrageux d'Adélard Lévesque. La disposition typographique, libérée de ponctuation et de majuscules, espace les groupes rythmiques et dégage les réflexions du récit principal et de ses descriptions. 
Perrault retient encore quatre sections qui, à l'origine, devaient précéder Gélivures: les premières versions, intitulées Terre à terre, Terre' à quai, Fleur de peau et Mer et monde, racontant l'exil des hommes en Abitibi, quelques souvenirs de la Côte Nord et le présent désœuvré des riverains. L'auteur risque encore de changer bien des choses dans l'ensemble avant de publier ces manuscrits, mais on peut s'attendre à ce que le deuxième volet de son long poème épique vienne agrandir davantage la dimension mythique de son œuvre.

\section{Poésies diverses}

En plus des deux œuvres majeures, Chouennes et Gélivures, quatre autres textes de Pierre Perrault méritent d'être cités.

Noël ancien dans une vallée nouvelle est un long poème en versets libres, rempli d'allusions à la forêt des bûcheroris et des chasseurs, à l'épinette noire transformée en arbre de Noël par les marchands, et recherchée jusqu'en ville par un lièvre, un lièvre témoin de la légende qui se perpétue dans le décor du rude hiver canadien. L'histoire raconte qu'un "homme d'entre-nous... / un homme-seul-avec-son-feu-et-la-toiled'araignée-de-ses-pistes" attend la naissance de son fils, et l'avenir le préoccupe; son épouse, “une femme à peine connue par l'homme»; "donne une neige à l'enfant". Et cet enfant, qu'il devienne charpentier de navires comme les gens de l'île-aux-Coudres ou prophète comme les poètes, “il lui suffira de peupler ce monde." L'histoire de la naissance de l'Enfant-Dieu inspire de l'espoir: cela peut se répéter. Et voilà la naissance d'un fils, dieu et soleil, espoir du peuple dans une vallée nouvelle qu'on devine traversée par un fleuve.

Les trois autres publications de Perrault ont pour objet les hommes de la ville de Montréal. Les textes rejoignent l'époque des émissions radiophoniques de l'auteur: "Imageries sur ma ville" (1961), "Ballades du temps précieux" (1961) et "J'habite une ville" (1965). Chanson des voyageurs du temps passé en vain est une ode et une élégie à la fois qui reprend une émission de la série radiophonique «Ballades du temps précieux" et raconte les vagabonds de la rue Saint-Laurent. Yves Lacroix a transcrit, en appendice à sa thèse, la dix-septième émission qui date du 6 mai 1961 et qui commence par «Que sont devenus les grands voyageưrs d'autrefois $^{15}$ ? . Dans le poème Chanson des voyageurs..., Pierre Perrault intercale des citations d'enregistrements faits sur la Main et parle pour la première fois du dénommé Lablague, transformé dans le poème dramatique J'habite une ville, en un soudeur qui grimpe au sommet des édifices de Montréal comme au grand mât d'un navire et y rêve de ciel et de mer. Le poème dramatique J'habite une ville laisse aussi parler un homme et une femme qui ont goût de pays dans cette ville qui est une île. En 1962, le Centre d'essai a créé J'habite une ville, à l'école des Beaux-Arts. En 1965, une publication tout à fait différente est parue dans le Devoir, sous le même 
titre; J'habite une ville, le reportage poétique, raconte la vie abrutissante des ouvriers de Montréal. L'auteur y glisse les paroles de Jean-Marc Joyal, né à Saint-David de Yamaska, et d'Étienne Bujold de Bonaventure, en Gaspésie, partis de chez eux à 14 et 15 ans, sans éducation, pour travailler. Ils ont quitté la terre que leur mère a dû vendre à l'encan et ils travaillent de neuf à cinq dans les abattoirs de la métropole,

dans une ville cadenassée, minutée,

horlogée, grignotée par les aiguilles;

poinçonnée, alarmée par les sirènes...

qui engloutit chaque matin à la

même heure, dans la mâchoire des

engrenages, des millions d'heures

de travail ${ }^{16}$

Perrault s'inquiète du sort de ces "locataires à demeure" dans la ville "de plus en plus machinale"; il dit la misère de ces exilés des grands espaces, hommes déchus qui n'inspirent plus de respect et qui se méprisent eux-mêmes. Mais malgré tout, dans le présent désespéré d'un peuple conquis, asservi, l'esprit prométhéen de la poésie de Perrault prend son essor et affiche à l'égard de la québécoisie une fierté pleine d'amour et de courage.

\section{La parole de Perrault}

Pierre Perrault réussit à élever au rang de l'esthétique la voix du peuple et la banalité du quotidien. Il allie à la puissance évocatrice d'une imagerie riche et fraîche, des tournures prosaïques qui opposent le sublime au vernaculaire et de ce fait révèlent parfois l'absurde tout en jetant un voile de légèreté sur le tragique des situations. Sa phraséologie volubile et sentencieuse exploite tout un réseau de symboles. Son vocabulaire marin et végétal s'enrichit de néologismes, d'expressions liturgiques et de termes juridiques, commerciaux et guerriers. Parfois un certain brio donne à la poésie un ton pompeux qui sonne faux: la verve désordonnée, les sons pour l'amour du son, étouffent quelques fois l'authenticité du sentiment. Parfois des constructions lourdes, l'absence de ponctuation, des pronoms équivoques rendent le sens ambigu et la forme un peu gauche. Mais en général, on peut admettre avec Van Schendel que

Si le langage manque d'une précision

de forme qui paraît regrettable, il se

nourrit ainsi d'une exactitude plus élevée,

celle véridique de l'esprit de poésie, qui fait

son feu de toute maladresse et brûle

au détour d'un mot riche d'expérience ${ }^{17}$

Le poète crée: il invente non seulement des images mais des mots. L'invention verbale de Perrault s'identifie surtout à la langue québécoise: "je jeunesserai»; «je me bilingularise»; «tu m'embâcles et je te débâ$\mathrm{cle}^{18}$. On peut affirmer, pour l'avoir entendu dire par l'auteur, que Perrault a inventé le substantif "grivolive ${ }^{19}$ " pour désigner la grive à dos olive. 
Si on ne peut jurer que Perrault soit le créateur de mots tels que "rivageries", "labradorite", “policiers ismatiques", ni qu'il ait été le premier à parler de "saxonie" et de "saxophone" pour désigner l'Anglais, on peut parler ici d'invention d'images nouvelles dans des verbes comme «je taillemer et fend-le vent... je tache d'huile ${ }^{20}$ ".

Le langage poétique de Perrault mêle les images des îles, de la mer, des arbres, du verger, des oiseaux, des bêtes et des saisons aux signes des hommes (maisons, lucarnes, armoires de pin jaune, navires) déjà vus dans ses films et marqués dans les poèmes par l'ampleur d'une symbolique qui évoque les sources de l'existence. Une foule de mots privilégiés mériteraient une analyse approfondie. Entre autres, "pylônes ${ }^{21}$ " à un moment donné, rappelle la séquence du film Un pays sans bon sens où Didier parle de mandat à remplir devant l'image des pylônes du projet hydroélectrique de la Manic: "pylônes" devient symbole de libre entreprise qui encourage la fierté, ou de projet collectif qui peut unir le peuple. Perrault parle aussi de "violon" et de «violoncelle 22 " et ces mots prennent selon le contexte le sens de musique, de matière aux rêves, de chemin ou d'obstacle. Les “loups", symboles de férocité, de sauvagerie parfois domestiquée, et les "lièvres", images de crainte, de silence et d'indécision, animent souvent la poésie de Perrault ${ }^{23}$. Dans Gélivures, les images des "harfangs" et des "lemmings" s'ajoutent aux symboles des oiseaux et des bêtes.

Les oiseaux sont une des sources de la poésie de Perrault. L'image de l'oiseau rejoint le thème de parole; l'oiseau est pensée qui a des ailes et cri qui porte loin. Et quand l'indignation délenche la colère du poète et provoque un réquisitoire désordonné, Perrault s'explique: "Il me monte à la gorge une rafale d'oiseaux qui brouille les ondes ${ }^{24}$." Perrault s'identifie à certains oiseaux: solitaire comme le huard, et éveillé comme le hibou, il habite le passé et le silence des siens. Sa parole est un message troublant, le chant d'un oiseau qui «prend par surprise», un «long cri de huard" qui "anéantit l'indifférence et les satisfactions prématurées ${ }^{25}$ ". Et la langue du peuple, les nombreuses traditions orales, les légendes et les exploits inédits, voilá une matièrè volatile que Perrault voudrait bien «réchapper":

de tous ces grands textes poussiéreux enfermés dans les conforts de l'écriture et l'aisance des syntaxes impérieuses qu'en est-il à peine une plume échappée au grand vol migratoire

allons-nous encore une fois reprocher aux oiseaux de n'avoir pas pris racine quand leur ame est volubile quand leur âme est volatile ${ }^{26}$

De la racine jusqu'au fruit, l'arbre est une autre source de symboles. 
Perrault exploite le langage des arbres. Dans Portulan d'abord, le. poème Arbre généalogique établit la métaphore de base qui rattache l'image au thème de l'appartenance et de "l'acharnation" comme dirait Alexis Tremblay de l'île-aux-Coudres. Les poèmes Vie à trépas, Écorce, Verger, l'Autre monde, Arborescence identifient le peuple au verger, le poète à "l'orme de septembre". Dans Ballades du temps précieux, l'allusion à un vent qui s'élève au cœur d'un verger peut évoquer l'espoir dans un éveil national ${ }^{27}$; une orange, fruit unique et isolé, disparaîtra mais le poète garde espoir dans le fait que "le verger... survit à ce monde de peu de parole ${ }^{28}$ ". Dans Toutes isles, l'image de l'arbre se mêle à d'autres métaphores pour parler du vent et de ses charmes, et du goût du voyage,

\section{... tentations qui pendent aux \\ branches du vent: quel arbre \\ c'était, plein de lèvres et sans \\ bouche ${ }^{29}$.}

Puis, la mer et les îles ont longtemps dominé dans le jeu poétique des symboles de Perrault. La mer, imprévisible et séduisante, lieu de tempête et de naufrages, invite toujours au voyage, à l'exploit. L'île sur la mer est un havre isolé, synonyme de sécurité et de solitude. Dans le poème dramatique J'habite une ville, Pierre Perrault, qui considère Montréal comme une île, présente une image marine des soudeurs. Les édifices en constructions sont des filets d'acier; les soudeurs, des «scaphandriers de la mer des ailes ». Le poète soutient jusqu'à la fin l'allégorie du ciel-mer et de l'édifice-arbre-et-mât:
... ma ville s'élève de plus en plus
haut sur des arêtes d'espace poissonneux...
... un mât, un mât nous élève
sans raison et à nos détriments
jusqu'à la hauteur des oiseau $x^{30}$.

Au langage de la mer s'accroche automatiquement le langage du départ, des navires et des naufrages. Dans un poème de Portulan, intitulé Alors, alors, alors, les mots clés s'enchaînent entremêlés aux paroles des chansons et de contes, aux expressions familières empruntées au vocabulaire marin: “le vent des désirs", "coffres d'épaves", "anneaux d'or", «ils ont tracé dans l'eau trouble / d'un port de mer et d'hommes / la prime bordée d'une légende au long cours ${ }^{31}$ ". Goélettes du fleuve, cordiers du temps présent, vaisseaux de Jacques Cartier ou bateaux du roi tant attendus à l'époque de la prise de Québec, ils sont souvent trois navires, dans la poésie de Perrault. Et parfois, il s'agit de chasse-galerie... Le départ évoque un éveil, une prise de conscience: «Un jour, lever l'ancre / du langage maternel ${ }^{\mathbf{3 2}}$ w. Il faut revoir le film les Voitures d'eau, relire les passages de Toutes isles où Perrault parle des épaves le long de l'île d'Anticosti ou de la naissance d'un canot, pour saisir toutes les émotions du poète lorsqu'il parle de bateaux et de naufrages, pour comprendre qu'il puisse personnifier les navires et trouver une affinité entre l'âme humaine et celle des barques. Perrault peut faire allusion aux mauvaises pêches et laisser 
croire à bien autre chose; il peut se comparer à un navire en quête d'avenir et de richesse, prêt à naviguer jusqu'à ce qu'il échoue. Navire, symbole d'œuvre créatrice, de projet national, promesse de voyage comme d'avenir; naufrage, symbole de destruction et de mort, espoir des pilleurs d'épaves.

\section{nous naufrageons sous les coups de stercoraires d'acier... ton éloquence à pied levé me sert d'épave à propos nous irons au bout de ce naufrage ${ }^{33}$.}

Perrault n'ignore pas non plus le symbolisme des saisons lié à celui du jour, de la nuit, de la neige. Le printemps c'est le temps de la débâcle, du renouveau; la fin des longues nuits, le temps des fleurs qui porteront fruits, le retour des oiseaux. Ces images servent de base au symbole de libération de tout le pays dans des poèmes comme le Cantouque des cantouques ou Fleur à feu.34. L'hiver par contraste est le temps du froid, de la solitude, du sommeil, de la mort, du rêve aussi. L'hiver symbolise ce temps de la vie «où les vieux n'ont plus droit à la parole" et où «peu à peu les mots se perdent sans laisser traces ${ }^{35}$ ". Les paroles, qui sont “eau " dans la poésie de Perrault, gèlent en hiver et c'est en juin que les mots sont libérés des ruisseaux ${ }^{36}$. La neige aux yeux du poète est rêve dont on parle. Et Perrault écrit dans Gélivures:

je confie à tes neiges

le soin de tomber à l'improviste

ma floconneuse

sur le royaume de plein droit ${ }^{37}$

Le langage religieux est une autre source d'images pour Perrault. Portulan abonde d'évocations bibliques chargées d'allusions au rachat d'un pays, à la libération d'un peuple, à la défense d'une langue. Dans Croix de chemin, la recherche de libération se traduit dans l'image d'un sauveur promis qui se féminise en chanson-poème ${ }^{38}$. Noël ancien dans une vallée nouvelle par son allusion au mystère de l'Incarnation, évoque la naissance de tout un pays. Parfois, Perrault ne peut s'empêcher de faire la morale au clergé. Le clocher et l'église sont symboles du clergé qui a longtemps dominé le peuple silencieux. Perrault y trouve des images remplies de tristesse:

nous sommes dans la mort comme l'église du village dans l'eau de la rivière ${ }^{39}$

Mes visages tombés du clocher dans l'eau de l'étang sont devenus carpes; elles ont vécu cent ans ${ }^{40}$

Dans Un poisson dans la mer et En désespoir de cause, Perrault reproche au clergé d'avoir endormi le peuple avec «un royaume qui n'est pas de 
ce monde", d'avoir encouragé "l'usage excessif de l'autre joue"; il lance des traits ironiques à la revanche des berceaux, au patron des innocents, Saint-Jean-Baptiste; il relie le langage du clergé au langage de l'économie en parlant de prébendes et de vendeurs du temple. D'un autre côté, il a de ces tournures qui résonnent comme un écho d'évangile; "alors, je vous le dis..." (Recréation). Des expressions religieuses côtoient des mots marins: "où vont échouer mes litanies" (l'Autre monde). Des termes religieux s'échappent dans de pieuses métaphores laïcisées en communion avec la nature: «ll se passe des choses capitales / dans le cloître des racines" (Migration) ${ }^{41}$. Dans Noël ancien dans une vallée nouvelle, Perrault décrit la neige "blanche et anachorète sur la bure des épinettes noires ${ }^{42}$ ", allusion à la solitude des étendues enneigées, et comparaison de l'écorce de l'épinette au vêtement de grossière étoffe brune que porte le moine. Et dans ce même poème, l'homme qui devient père s'associe au prêtre:

Avec des mots les plus chair, les plus sang, les plus pain et vin et fromage, avec des mots, il a disposé de l'ombre et honoré les vents du nord ${ }^{43}$

Pour qui admet la puissance surnaturelle des paroles du prêtre au moment de la transsubstantiation, il y a ici une très belle transposition des paroles toutes simples d'un homme ordinaire - le roturier se réfugie dans le mot "fromage" - en un parolis merveilleux qui opère un mystère comparable à la consécration. De même, on ne peut passer sous silence la puissante image eucharistique dans À poing fermé : "Les deux poings de la colère / qu'il faut bénir et rompre / et qu'il faut partager ${ }^{44}$ "; notons aussi l'allusion biblique à l'arbre de connaissance, qui se mêle à l'image du Québec, ce pays à l'odeur de pomme et de cidre ${ }^{45}$ (Fruit défendu, Optique, Angoisse). Enfin, il y a toute l'ampleur du mot "Verbe" et du mot "Parole" qu'on associe aux évangiles. Pierre Perrault prophétise l'avènement d'un sauveur avec la résonance de l'évangile de saint Jean dans le poème Patrimoine, d'abord, puis dans Noël ancien... : Perrault évoque, dans ce dernier, l'héroïsme du peuple, "tant et tant de chair qui veut se faire verbe et habiter entre nous". Cette chair est matière à transformer en récit épique pour la fierté et l'orgueil du pays.

Dans la présentation d'En désespoir de cause, l'emploi d'un vocabulaire juridique, commercial et martial a déjà été signalé. Il suffit d'ajouter que Perrault transpose une parole vécue dans une forme que certains trouvent ambiguë mais qui crée une atmosphère ésotérique propre au prestige du mythe. II y a par exemple, mélange de termes oniriques, juridiques et astrologiques, pour marquer l'importance d'une prise de conscience, malgré le goût de la sécurité que le peuple retrouve dans ses rêves, son silence et ses proverbes défaitistes:

... la lune compte plus que le soleil

dans les solstices alors le songe

se confie à la chaleur des proverbes... 
... Il nous reste à naître un soleil de

première instance. Un homme aussi

entend minuit qui répond du soleil

Alors donc, il a pensé le soleil à pleines

mains au sommet des cadrans solaires ${ }^{46}$.

II y a convergence de langages des affaires, des arbres et du sang, pour marquer l'inquiétude que suscite la prise de conscience et l'agitation nationale au Québec: "L'indice de turbulence s'en rapporte à l'accélération de la sève dans les veines du marbre des statues encore en bois debout ${ }^{47}$. " La difficulté que pose la langue poétique de Perrault découle de la confusion des langages qui nourrissent ses visións énigmatiques. On pourrait parler longuement du langage des astres, du symbolisme de la nuit, et des allusions nombreuses au sang dans la poésie de Perrault:

Mais le langage de prédilection dans les poèmes de Pierre Perrault, est celui du peuple. Les paroles des Acadiens, des gens de la Côte Nord, des journaliers montréalais, mots pleins d'expérience, chouennes et dictons, le poète les aime et les répètent comme un exorcisme contre le temps, l'histoire, le mercantilisme et la haute littérature qui ne savent même pas qu'ils sont à la veille de tuer une langue. Parmi les expressions populaires les plus fréquentes dans la poésie de Perrault, on note des compléments et des locutions comme "à vrai dire", "à outrance", "à fleur de peau», «à petit feu», «la rage aux dents», "du cceur au ventre», «du plomb dans l'aile». Ce qui surprend le lecteur et ajoute à la puissance évocatrice de ces tournures c'est leur accumulation et leur juxtaposition inusitée: «...qu'on me prenne au mot, à la gorge, au tragique et à la claire fontaine ${ }^{48}$ ". Parmi les expressions à résonance mythique qui donnent toute son ampleur à la verve de Perrault, relevons: "tout se passe comme si...", expression familière et généralisation à la fois, qui tend à mystifier, à donner l'impression qu'il existe des forces auxquelles tout est soumis; «alors... alors... alors", répétition qui marque un haut degré d'excitation dans le récit ou annonce une nouvelle; "pourtant... pourtant", répétition qui annonce ou sous-entend les faits qui justifieraient une autre tournure des événements; la tournure superlative "plus que», tel que dans "fille plus que fontaine", «il est plus que temps", qui marque l'insistance ou exprime le dépassement; "un jour... un jour..." répétition qui révèle l'obsession du royaume comme dans les textes de Gélivures.

La phrase de Perrault exploite l'effet rythmique de l'énumération accouplée à la répétition, soit dans un enchaînement d'idées, soit dans une suite époustouflante de courtes propositions indépendantes à la première personne. Le poète intervertit l'ordre des mots et change parfois une partie de l'expression; ce procédé joue sur le poids des mots et, comme un refrain, la répétition crée musique et insistance:

j'ai peur d'être trop étroit

au soir du grand jour

j'ai peur d'être un peu sourd

le jour du grand soir ${ }^{49}$ 
Les nombreuses subordonnées adjectives dans les poèmes de Perrault personnifient souvent les choses qu'il décrit; "des, silences qui en disent long "; des amours, des rêves "qui n'en croient pas leurs yeux". Les interrogatives, "Qui?... Qui dira...?" ou "Allons-nous...?", marquent d'une certaine véhémence la préoccupation du poète et cherchent l'adhésion de tous ceux qui sont pris à témoin. Perrault exploite l'équilibre des constructions ternaires et les techniques du refrain, jouant avec le son, signifiant l'insistance. La phrase de Perrault, en somme, reste simple et n'emploie pas souvent l'inversion. Son verbe joue surtout à l'indicatif, à tous les temps de la langue parlée. L'impératif y a sa place. Et les infinitives et les exclamatives savent varier le rythme.

"Familier" est un nom ou un adjectif qui désigne un ami intime, une chose bien connue, une expression naturelle ou encore une attitude désinvolte. Perrault surprend le lecteur en évitant toute ces significations: dans Portulan, Familier est le titre d'un poème sur la femme. Le calembour vient du son «fam».

"Cornouailles» existe qu'en tant que nom propre traduisant «Cornwall ", comté maritime au sud-ouest de l'Angleterre. Dans Gélivures, ce n'est certes pas le sens du mot: le poète joue avec des sons tels que "cornes» et «fiançailles» pour inventer le titre d'un poème sur les bœufs musqués qui se disputent une harde de femelles.

Les jeux de mots continuent dans des surprises antonymiques comme "le château-faible de mon âme". Il y a parfois substitution de consonnes telle que "les fruits nous font tourner / la terre"; ou substitution d'une syllabe atone par une syllabe accentuée comme “j'ai donné à mes regrets / des chansons de se réjouir 50 ". Perrault module des variations à partir de chansons de folklore (Isabeau s'y promene), de contes (Anne, ma sceur Anne) et de fables (le Chêne et le roseau). II cite des proverbes en ajoutant un commentaire lapidaire; il fabrique ou déforme des aphorismes. L'esprit qu'il y manifeste donne à ses dires un ton mystificateur, sage ou prophétique: "la faim justifie les moyens"; "ventre affamé n'a pas d'oreilles et j'ai un royaume dans les talons ${ }^{51}$ ".

Si Perrault invente beaucoup, il décore aussi son cuvre poétique de paroles qu'il aime. II insère quelquefois dans ses poèmes des bribes de conversations en langue vernaculaire. Ces paroles d'un homme du peuple viennent alors appuyer les dires du poète ou bien elles sont le cœur du récit. Dans Chanson des voyageurs du temps passé en vain, Perrault cite un dénommé Lablague, enregistré sur la Main et il parle ensuite des mains de ce voleur comme il a parlé dans Toutes isles, des mains montagnaises, inspirant le respect et l'admiration malgré la déchéance. Quant Perrault laisse parler Étienne Bujold ou Jean-Marc Joyal dans le reportage J'habite une ville, c'est pour appuyer ses récriminations contre la misère d'un peuple de manceuvres et de locataires. Quand il reprend les paroles d'Arthur Côté dans Neigeries, Perrault veut rendre hommage à “celui qui s'excuse de n'avoir pas avec ses deux mains nues réussi le royaume proposés2 
Ce qui caractérise l'écriture de Pierre Perrault c'est son souffle épique à saveur québécoise et contemporaine. La parole de Perrault s'applique à dire cette terre qu'il aime, le fleuve, le roc, la faune et la flore de tout ce territoire qui l'émerveille, qui nourrit sa poésie d'images réalistes et transcendantes à la fois et qui forme un cadre épique à la mesure des héros en quête d'un pays.

L'œuvre poétique de Pierre Perrault demeure une entreprise d'orgueil qui incorpore la multitude des archétypes à sa disposition, dans un monde tangible, réel et présent; et cela depuis les légendes de Toutes isles jusqu'au combat taurobolique de Gélivures, à travers les "parlêmes " de J'habite une ville et de la Chanson des voyageurs du temps passé en vain, et à travers la convergence ésotérique des langages de Chouennes et de Noël ancien dans une vallée nouvelle. On pourrait encore analyser la valeur poétique des œuvres de Perrault dans tous les domaines, radio, théâtre, cinéma et les découvrir imprégnées d'une même poésie, d'esprit mythique et québécois.

Jocelyne Tessier

1. Portulan, Prix du Grand Jury des lettres canadiennes, 1961. Chouennes, Prix du Gouverneur général, 1975.

2. Pierre Perrault, Gélivures, Montréal, Hexagone, 1977, 209 p.

3. Id., Chouennes, Montréal, Hexagone, 1975, 317 p.

4. Id., Toutes is/es, Montréal, Fides, 1963, 189 p. Des extraits de Toutes isles sont parus dans Cahiers d'essai, Montréal, éd. d'Essai, $n^{\circ} 3$, janvier 1961 , p. 25-31. En 1967, il y eut deuxième édition de Toutes is/es, en livre de poche. La première édition, véritablement artistique mérite l'éloge de Pierre Pagé, dans Lectures, juin 1963, p. 262 : "Toutes is/es... est d'ailleurs bien servi par la magnifique présentation que les éditions Fides lui ont donnée. D'un format large, aéré, digne du grand souffle poétique de ces pages, l'ouvrage est enrichi de huit hors-texte en couleurs, ceuvres de l'auteur lui-même. "

5. Id., Noël ancien dans une vallée nouvelle, dans le Devoir, Montréal, vol. 53, $n^{\circ} 300$, le 22 décembre 1962, p. 9, 11.

6. Id., Chanson des voyageurs du temps passé en vain, dans Lettres et écritures, vol. $1, \mathrm{n}^{\circ} 1$, décembre 1963, p. 21-30.

7. Id., J'habite une ville, poème dramatique, dans Liberté, vol. $5, n^{\circ} 4$, juillet-août, 1963 , p. 375-384; J'habite une ville, reportage poétique, dans le Devoir, . Montréal, vol. $56, n^{\circ} 143$, le 19 juin, 1965, p. 31.

8. Jean Marcel, "Pierre Perrault, poète", dans l'Action nationale, Montréal, vol. 54 , $n^{\circ} 8$, avril 1965, p. 820.

9. Pierre Perrault, Toutes isles, p. 71.

10. Gilles Marcotte, Toutes isles, dans la Presse, Montréal, vol. $79, n^{\circ} 211$, le 22 juin 1963, supplément, p. 8.

11. Pierre Perrault, Chouennes, p. 7. 
12. Ibid., p. 190.

13. Id., Gélivures, p. 189.

14. Pierre Deffontaines, l'Homme et l'hiver au Canada, Paris, Gallimard, 1957, p. 25.

15. Yves Lacroix, Pierre Perrault, Poète de la parole, thèse de maîtrise présentée à la Faculté des lettres de l'Université de Montréal en 1972, appendice, p. 115132.

16. Pierre Perrault, J'habite une ville, dans le Devoir, p. 31.

17. Michel Van Schendel, Livres et auteurs. canadiens 1961, Montréal, Éditions de Jumonville (Les Presses de l'Université Laval), 1962, p. 34.

18. Pierre Perrault, Chöuennes, En désespoir de cause, p. 210, 212, 230.

19. Ibid., p. 245.

20. Ibid., p. 209.

21. Ibid., p. 234.

22. Id., Chouennes, p. 44, 75, 135, 225, 227, 295, 297.

23. Id., Chouennes, p. 207, 242; Noël ancien dans une vallée nouvelle; Gélivures, p. $37,54,57,70,73,183$ (loup) ; p. 47, 94, 127, 173 (lièvre).

24. Id., Chouennes, p. 225.

25. Ibid., p. 80-81.

26. Id., Gélivures, p. 191-192.

27. Id., Chouennes, p. 153, 158-159, 162-163, 192-193.

28. Ibid., p. 191.

29. Id., Toutes isles, p. 62.

30. Id., J'habite une ville dans Liberté; p. 384.

31. Id., Chouennes, p. 130.

32. Ibid., p. 18.

33. Ibid., p. 233.

34. Ibid., le Cantouque, p. 224-236; Fleur à feu, p. 60-61.

35. Id., Toutes isles, p. 161.

36. Id., Chouennes, p. 187.

37. Id., Gélivures, p. 201.

38. Id., Chouennes, p. 124.

39. Ibid., p. 12.

40. Ibid., p. 39.

41. Ibid., p. 108, 24, 13.

42. Id., Noël ancien dans une vallée nouvelle, p. 9.

43. Ibid., p. 11.

44. Id., Chouennes, p. 64.

45. Ibid., p. 30, 40, 79.

46. Id., Noël ancien dans une vallée nouvelle, p. 9.

47. Id., Chouennes, p. 214.

48. Ibid., p. 203.

49. Ibid., p. 142; cf aussi p. 242-247.

50. Ibid., p. $108 ; 136 ; 162$.

51. Ibid., p. $210 ; 217$.

52. Id., Gélivures, p. 36 